Submitted to MRS Proceedings, Volume 629, Interfaces, Adhesion and Processing in Polymer Systems, 2000

\title{
The Effect of Surface Contamination on Adhesive Forces as Measured by Contact Mechanics
}

\author{
John A. Emerson, Rachel K. Giunta, Gregory V. Miller, Christopher R. Sorensen, and \\ Raymond A. Pearson ${ }^{1}$ \\ Department of Organic Materials, Sandia National Laboratories, \\ Albuquerque, NM 87185-0958, USA \\ ${ }^{1}$ Materials Science and Engineering Department, Lehigh University, \\ Bethlehem, PA 18015-3195, USA
}

\begin{abstract}
The contact adhesive forces between two surfaces, one being a soft hemisphere and the other being a hard plate, can readily be determined by applying an external compressive load to mate the two surfaces and subsequently applying a tensile load to peel the surfaces apart. The contact region is assumed the superposition of elastic Hertzian pressure and of the attractive surface forces that act only over the contact area. What are the effects of the degree of surface contamination on adhesive forces? Clean aluminum surfaces were coated with hexadecane as a controlled contaminant. The force required to pull an elastomeric hemisphere from a surface was determined by contact mechanics, via the JKR model, using a model siloxane network for the elastomeric contact sphere. Due to the dispersive nature of the elastomer surface, larger forces were required to pull the sphere from a contaminated surface than a clean aluminum oxide surface.
\end{abstract}

\section{INTRODUCTION}

We are evaluating work of adhesion measurements to facilitate our understanding of adhesive failure mechanisms for systems containing encapsulated and bonded components. One issue under investigation is the effect of organic contamination on the adhesive strength of several polymer/metal interface combinations. We are interested in quantifying the level of contamination at which adhesive strength decreases. We have applied contact mechanics, the JKR method, to study this question. The model contaminant is hexadecane - a non-polar, medium molecular weight hydrocarbon fluid. We choose hexadecane because it replicates typical machining fluids, is nonreactive with aluminum oxide surfaces, and should not dissolve readily into the adhesive systems of interest. The application of a uniform, controllable and reproducible hexadecane layer on aluminum oxide surfaces has proven to be difficult [1]. A primary concern is whether studies of model systems can be extended to systems of technological interest.

The JKR theory, developed by Johnson, Kendall, and Roberts [2], is a continuum mechanics model of contact between two solid spheres. The JKR theory is an extension of Hertzian contact theory [3] and attributes the additional increase in the contact area between soft elastomeric hemispheres to adhesive forces between the two surfaces. The JKR theory allows a direct estimate of the surface free energy of the interface as well as the work of adhesion $\left(W_{a}\right)$ between solids. Early studies performed in this laboratory involved the determination of $W_{a}$ between silicone (PDMS) and aluminum surfaces in order to establish the potential adhesive failure mechanisms. A model PDMS elastomer and polymer treatments were developed for studying 


\section{DISCLAIMER}

This report was prepared as an account of work sponsored by an agency of the United States Government. Neither the United States Government nor any agency thereof, nor any of their employees, make any warranty, express or implied, or assumes any legal liability or responsibility for the accuracy, completeness, or usefulness of any information, apparatus, product, or process disclosed, or represents that its use would not infringe privately owned rights. Reference herein to any specific commercial product, process, or service by trade name, trademark, manufacturer, or otherwise does not necessarily constitute or imply its endorsement, recommendation, or favoring by the United States Government or any agency thereof. The views and opinions of authors expressed herein do not necessarily state or reflect those of the United States Government or any agency thereof. 


\section{DISCLAIMER}

Portions of this document may be illegible in electronic image products. Images are produced from the best available original document. 
solid surfaces by measuring the degree of self-adhesion hysteresis [4] as indicator of surface properties.

The goal of this work is to measure the adhesion between PDMS and aluminum surfaces as received, cleaned by two techniques, and contaminated with hexadecane. A custom-built JKR apparatus is used to determine the amount of hysteresis and $W_{a}$.

\section{EXPERIMENTAL}

\section{Preparation of PDMS hemispheres}

Model networks of poly(dimethylsiloxane) were synthesized by reacting vinyl-capped poly(dimethylsiloxane) with tetrakis (dimethylsiloxy) silane in the presence of a platinum catalyst. The use of the platinum-divinyltetramethyldisiloxane complex allowed the polymerization to proceed at room temperature without the formation of by-products. All reactants were used as received from Gelest Inc.

The procedure used to synthesize the PDMS networks is as follows. First, tetrakis (dimethylsiloxy) silane was added using an Eppendorf volumetric dispenser. With the amount of cross-linker fixed, a 0.75 stoichiometric ratio of vinyl-capped PDMS $(6000 \mathrm{~g} / \mathrm{mol})$ was added with a $3 \mathrm{ml}$ syringe. The contents were then mixed thoroughly and stored in a freezer at $-20^{\circ} \mathrm{C}$. Once the temperature was low enough to slow the reaction, approximately $150 \mathrm{ppm}$ of platinumdivinyltetramethyldisiloxane complex was worked into the system. Hemispheres, which are used as the JKR lens, were prepared using an oiler (medium, DL-32). Drops of the mixture were placed on tridecaflro-1,1,2,2-tetrahydroctyl trichlorosilane treated glass slides.

\section{Substrate preparation}

Polished Al 6061-T6 substrates, used in initial studies, proved too rough for determining film thickness of the hexadecane. Also the JKR measurements were unreliable because the roughness interfered with the contact area measurements. The average surface roughness, as determined by Dektak Surface Profilometer, measured 250 - $850 \AA$. All data reported here used substrates made by sputtering $400 \mathrm{~nm}$ of $\mathrm{Al}$ onto glass slides. The average roughness was $30 \AA$. The average hexadecane thickness, as measured by ellipsometry, was 10-20 $\AA$. Two cleaning procedures were applied: 1) acetone rinse followed by argon plasma clean; 2) acetone rinse followed by chromic-sulfuric clean/etch and HPLC grade water rinse. A clean $\mathrm{N}_{2}$ stream removed any residual water.

\section{JKR studies}

A hemispherical PDMS lens is mounted into the JKR apparatus. Measurements were made at room temperature and $30-60 \% \mathrm{RH}$. Loading and unloading measurements were done with an incremental change in displacement of $3 \mu \mathrm{m}$ per step. The work of adhesion was determined by recording the contact radius as a function of contact load and using the JKR equation to fit the data:

$$
\begin{aligned}
& a^{3}=(R / K)\left\{P+3 \pi W_{a} R+\left[6 \pi W_{a} R P+\left(3 \pi W_{a} R\right)^{2}\right]^{1 / 2}\right\}
\end{aligned}
$$

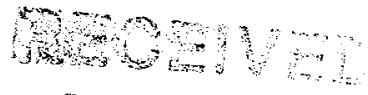

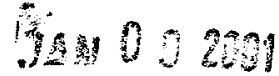


where $a$ is the contact radius, $R$ is the effective radius of the lenses, $K$ is the material property, $P$ is the contact load, and $W_{a}$ is the work of adhesion.

\section{RESULTS AND DISCUSSION}

An ideal JKR plot of the contact between two PDMS hemispheres [4] is illustrated in Figure 1. No hysteresis or negative tensile forces are observed, allowing accurate calculation of $W_{a}$ from Equation 1. In studying systems that have strong adhesive forces, the curves can be difficult to interpret because of nonlinearity in the unloading curves. Inconsistent JKR data was obtained from experiments involving PDMS hemispheres pressed against as-received sputtered aluminum substrates. A representative curve is shown in Figure 2. We found some form of cleaning was necessary. Two cleaning methods were investigated: chromic-sulfuric acid clean and argon plasma clean. Both methods of cleaning reduced the water contact angle from greater than $60^{\circ}$ to approximately $0^{\circ}$, indicating that organic contaminants had been removed. In Figure 3 , the JKR

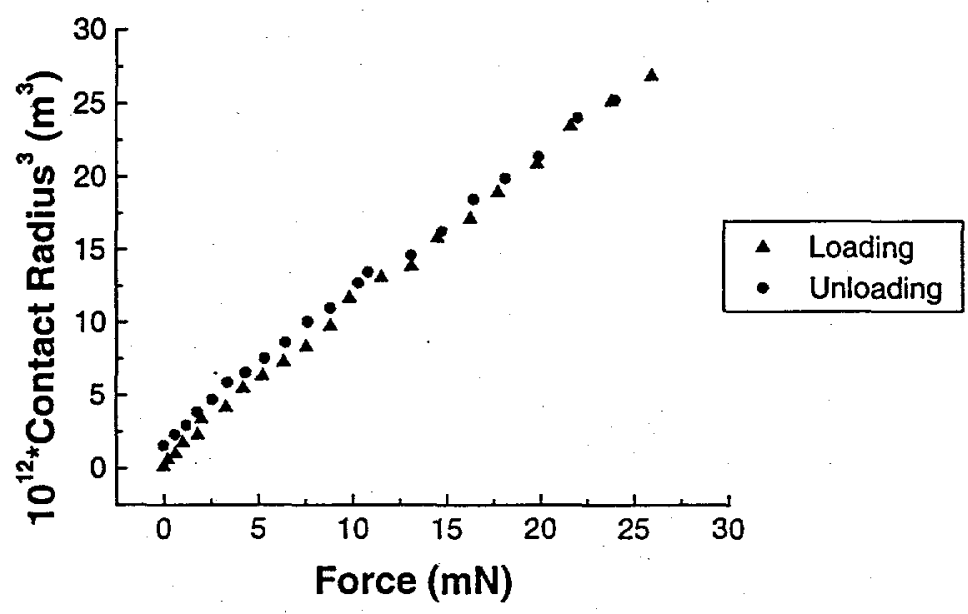

Figure 1. JKR plot of two PDMS hemispheres pressed together $W_{a}=44.02, K=1.06 x 10^{6}$ from Equation 1.

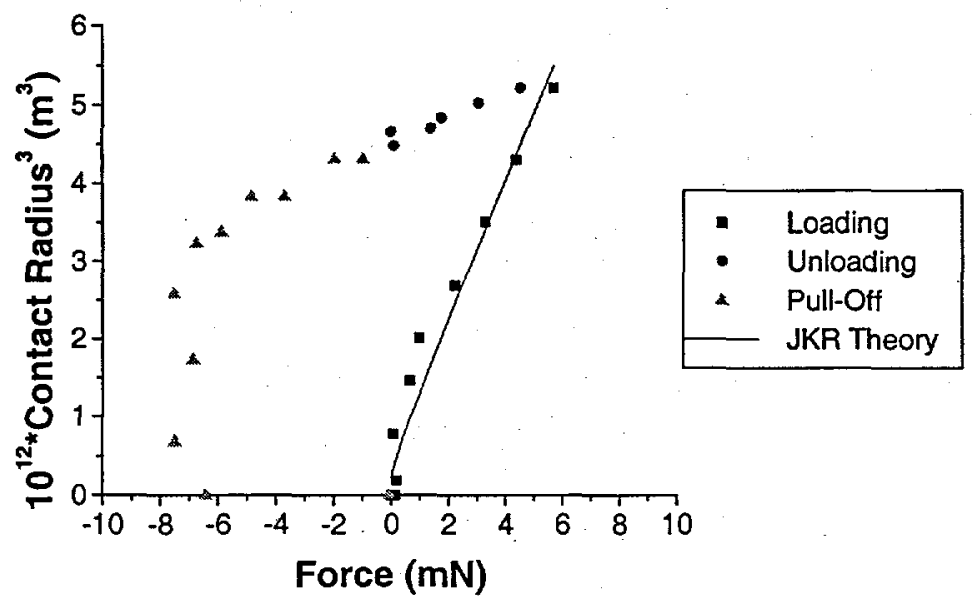

Figure 2. JKR plot of PDMS pressed against an as-received sputtered aluminum substrate. 
plot of PDMS pressed against chromic-sulfuric acid cleaned aluminum, a small amount of hysteresis and pull-off force is seen. A similar plot is obtained after cleaning with argon plasma, Figure 4. While it was initially expected that removal of organic contaminants would increase the surface interactions and thus increase the pull-off forces, this apparent anomaly is explained by analyzing the polar and dispersive components of the adhesive forces being probed. The surface energy of the PDMS was measured to be $22 \mathrm{~mJ} \mathrm{~m}^{-2}$ and is mostly non-polar. Thus, the pull-off forces measured are due to dispersive, not polar, surface interactions. The surface of the sputtered aluminum substrates, before and after cleaning, is aluminum oxide. The surface energy of aluminum oxide is mainly polar: the polar component is $538 \mathrm{~mJ} \mathrm{~m}^{-2}$ and the dispersive component is $100 \mathrm{~mJ} \mathrm{~m}^{-2}$. Organic contaminants, however, are mainly non-polar and thus would interact more strongly with the PDMS hemispheres.

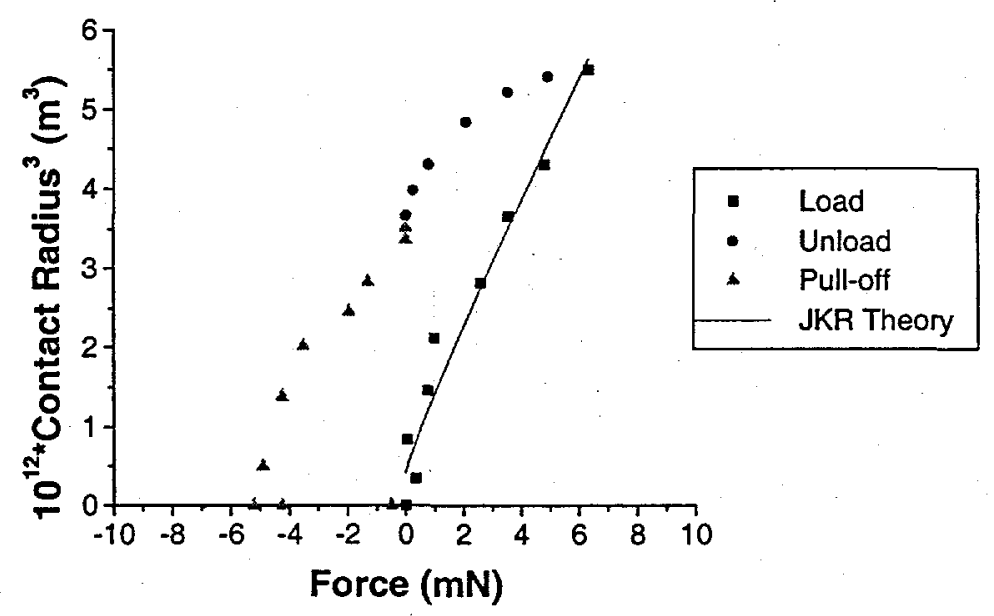

Figure 3. JKR plot of PDMS pressed against a sputtered aluminum substrate cleaned with chromic sulfuric acid.

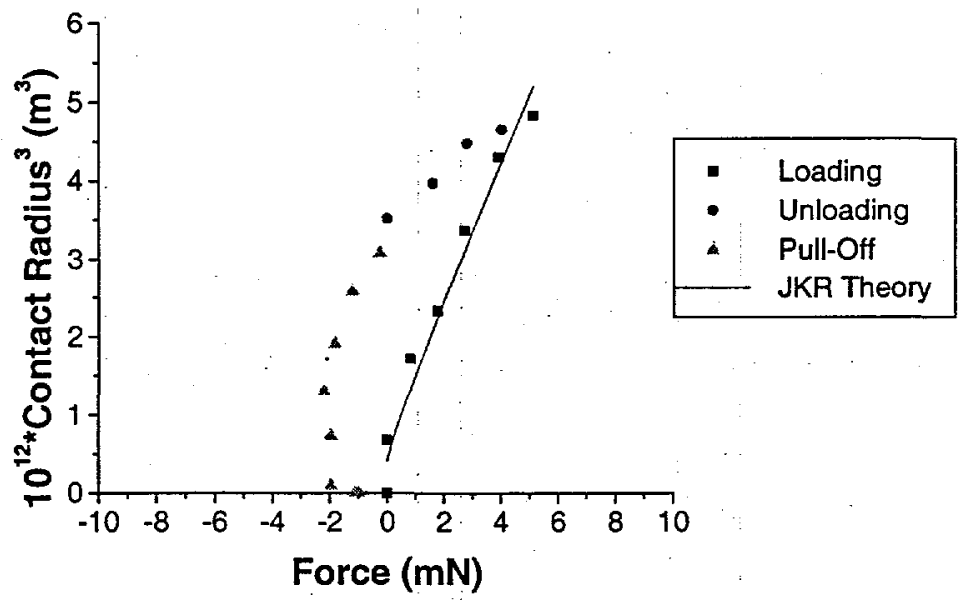

Figure 4. JKR plot of PDMS pressed against a sputtered aluminum substrate cleaned with argon plasma. 
To confirm that the change in pull-off forces after cleaning the sputtered aluminum substrates arose from the removal of organic contaminants rather than a change in the oxide structure, scanning electron micrographs were taken of the oxide surfaces before and after cleaning with chromic-sulfuric acid. As illustrated in Figure 5, there is no perceptible change in the texture of the aluminum oxide surface after the acid etch.

To investigate the interaction of PDMS with a controlled surface contaminant, a uniform layer of hexadecane was applied to clean aluminum substrates. The surface energy of hexadecane is primarily dispersive, and water contact angles of substrates contaminated with hexadecane were approximately $90^{\circ}$. A JKR plot of PDMS pressed against a chromic-sulfuric acid-cleaned substrate contaminated with hexadecane is given in Figure 6. The corresponding plot of the plasma-cleaned, hexadecane-contaminated substrate is shown in Figure 7. As with the asreceived substrates, pull-off forces were higher than those observed on the respective acidcleaned and plasma-cleaned substrates without hexadecane contamination.
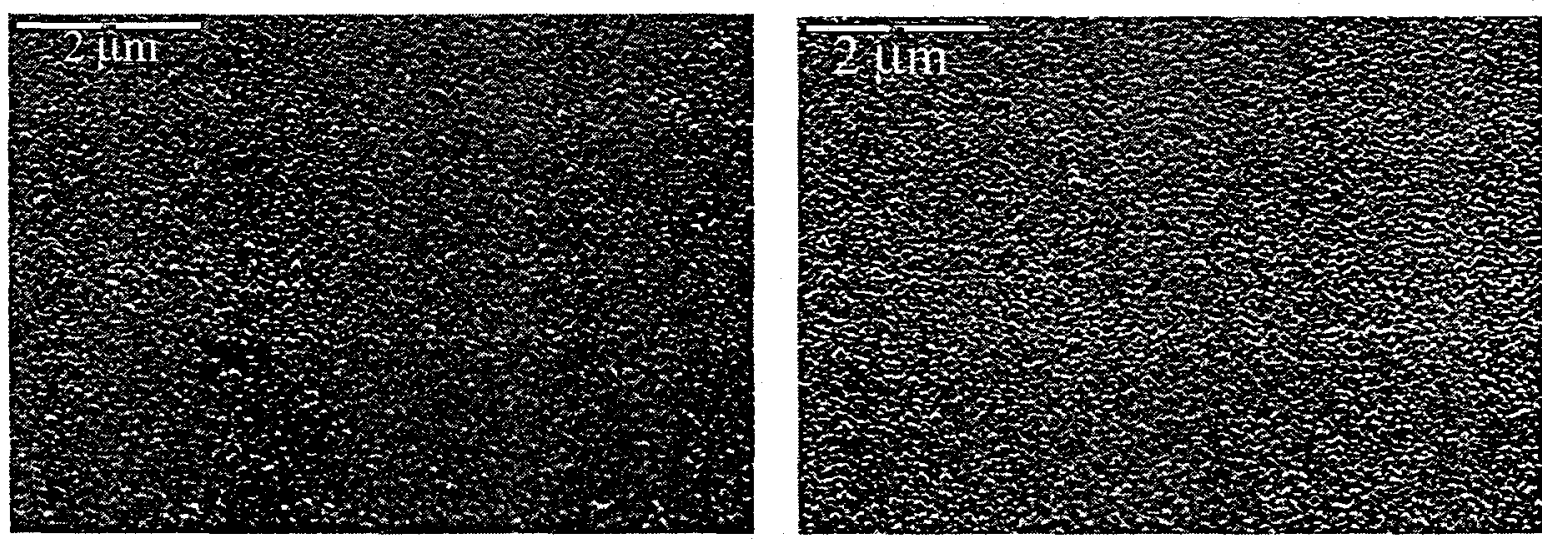

Figure 5. Scanning electron micrographs of sputtered aluminum substrate surfaces. As received, left. Chromic sulfuric acid cleaned, right.

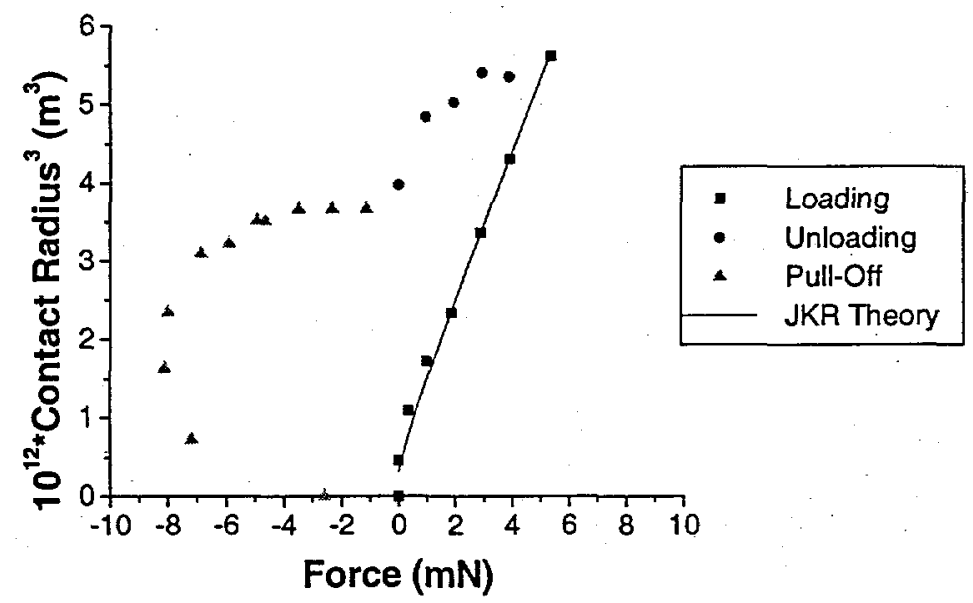

Figure 6. JKR plot of PDMS pressed against a sputtered aluminum substrate cleaned with chromic sulfuric acid and contaminated with hexadecane. 


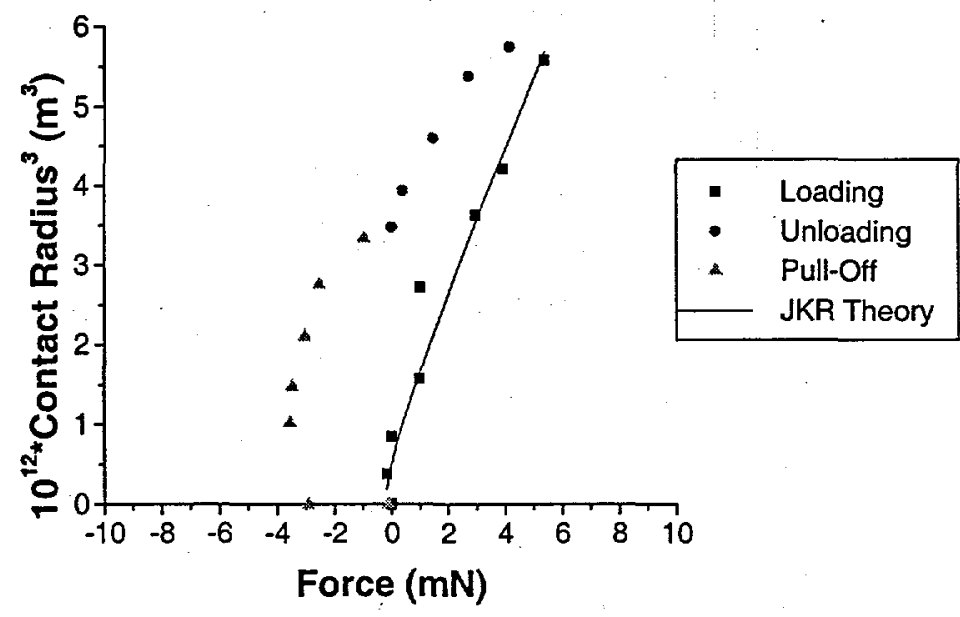

Figure 7. JKR plot of PDMS pressed against a sputtered aluminum substrate cleaned with argon plasma and contaminated with hexadecane.

\section{CONCLUSIONS AND FUTURE WORK}

The pull-off forces of a PDMS hemisphere pressed against a sputtered aluminum substrate varied with the degree of contamination of the aluminum substrate. Since dispersive forces dominate the PDMS surface energy, interactions with non-polar contaminants are stronger than the interactions with the polar aluminum oxide surface. This contact mechanics approach may prove to be an effective tool to analyze the effects of surface contaminants on adhesive bonding. Future work in this area will include probing the polar interactions at the substrate surface using an elastomeric epoxy hemisphere rather than PDMS.

\section{ACKNOWLEDGEMENTS}

This work was performed at Sandia National Laboratories, which is supported by the United States Department of Energy under contract number DE-AC04-94AL85000. Sandia is a multiprogram laboratory operated by Sandia Corporation, a Lockheed Martin Company, for the United States Department of Energy. We thank Michael L. Thomas for taking the ellipsometry measurements and Catharine $\mathrm{H}$. Sifford for determining surface roughness. In addition, we thank Carol Jones Adkins for helpful discussions.

\section{REFERENCES}

1. E. P. Lopez (private communication) Sandia National Laboratories, 1999.

2. K. L. Johnson, K. Kendall and A. D. Roberts, Proc. R. Soc. London: Part A, 324, 301 (1971).

3. H. Hertz, Gesammelte Werke, Leipzig, 1895.

4. J. A. Emerson, G. V. Miller, C. R. Sorensen, and R. A. Pearson, ACS Polymeric Materials:

Science and Engineering, 81, 385 (1999). 